\title{
MOUVEMENTS DES TROMPES ET PROGRESSION DES EUFS CHEZ LA BREBIS
}

\author{
S. WINTENBERGER-TORRES \\ Avec la collaboration technique de Micheline GÉrard. \\ Station de Recherches de Physiologie animale, \\ Centre nátional de Recherches zootechniques, Jouy-en-Josas.
}

\section{SOMMAIRE}

Nous avons étudié Ia motilité des trompes utérines, et leur rôle dans la descente des oufs, grâce à des enregistrements cinématographiques à vitesse lente ( 2 à 3 images/seconde).

L'étude a été effectuée in vitro et in vivo pour l'isthme et seulement in vivo pour l'ampoule, la plupart des mouvements musculaires disparaissant dans l'ampoule isolée.

En plaçant dans la portion de trompe étudiée soit des ovocytes colorés, soit des particules de matière plàstique noire de même taille, soit encore du carbone en suspension dans le Locke, on a pu mettre en évidence l'existence dans l'ampoule de contractions péristaltiques lentes qui assurent la progression vers l'isthme de l'ovocyte fraîchement pondu, donc entouré en tout ou partie de ses cellules coronaires. Dans l'isthme se manifestent pendant les 48 à 60 heures qui suivent l'ovulation, des mouvements antipéristaltiques puissants qui maintiennent l'œuf à la jonction isthme-ampoule. Ce sont les mouvements péristaltiques, intéressant soit l'isthme dans sa totalité, soit seulement des fractions de l'isthme, qui entraînent finalement l'ovocyte dans la corne utérine.

Les différents types de contractions ont pu être déclenchés expérimentalement en faisant varier l'équilibre oestrogène-progestérone chez des brebis castrées.

On sait que la durée du transit des œufs dans 1'oviducte est sensiblement de 3 jours chez les Mammifères. Ce transit s'effectue par étapes : 1'œuf franchit rapidement l'ampoule, puis reste pendant 2 jours ou plus à la base de celle-ci et traverse 1'isthme en moins de 24 heures. Ceci est particulièrement net chez la brebis (WINTENBERGER, I955).

Si ce schéma est généralement admis, par contre aucune explication satisfaisante des mécanismes mis en ouvre pour assurer cette progression discontinue n'a jamais été donnée; les contractions musculaires aussi bien que les mouvements ciliaires ou les courants liquides ont été successivement ou simultanément invoqués. Nous donnerons dans un mémoire ultérieur une analyse complète de la bibliographie sur cette question.

Rappelons seulement que, jusqu'ici, pour l'étude de la motricité des trompes 3 genres de techniques différentes ont été utilisées : 
- enregistrement graphique des contractions musculaires de la trompe en survie dans du Tyrode oxygéné (femme, de la FuENTE; I949-I950);

- étude (méthode de Rubin) des trompes insufflées au gaz carbonique et enregistrement in vivo des variations de pression manométrique (femme: SALMon et Mintz, I938; Bernstein et Ferester, i940; Davies, I948; Geistes; lapine : WHIMPFHEIFER et FORESTEN, I939).

- technique de la fenêtre abdominale sur un animal anesthésié ; cette méthode est uniquement subjective (truie: SECKINGER, I923; lapine : WESTMAN, I926).

Nous avons limité notre étude principalement au rôle des contractions musculaires et, compte tenu des vitesses de passage, différentes dans les 2 parties de la trompe, étudié séparément l'ampoule et l'isthme.

\section{MATÉRIEL ET MÉTHODES}

Toutes les brebis utilisées appartiennent à la race Ile de France.

Le début de l'œestrus est déterminé avec précision en utilisant trois fois par jour des béliers boute-en-train. De plus, chaque brebis reçoit en début d'osstrus 800 I. I. d'hormone gonadotrope sérique afin de provoquer l'ovulation 24 heures plus tard (OrTavant, Thibault et WinThenierger, 1949).

\section{A) ÊTUE in vivo.}

Les animaux, à jeun depuis 24 heures, sont anesthésiés avec du chloral et du protoxyde d'azote.

$$
\text { I. Ampoule. }
$$

La vitesse de descente a été étudiée grâce :

- soit à des macrospores de Sélaginelle introduites dans chacune des trompes d'une brebis;

- soit à des billes de matière plastique noire ayant la taille des ceufs de brebis ;

- soit à des paquets d'œufs de lapine fraîchement ovulés, imprégnés de carbone et introduits dans chacune des trompes d'une brebis.

Mais, tandis qu'un côté est replacé in situ, l'autre est maintenu à la surface de l'ouverture médio-ventrale pratiquée dans l'abdomen pour accéder aux organes génitaux. Cette trompe soumise à des observations et filmée pendant I heure, est régulièrement humidifiée, alternativement avec du Locke ou de l'eau distillée pour éviter une élévation localisée de la pression osmotique due à l'évaporation.

La trompe maintenue à l'extérieur est placée sur un support lui-même solidaire de la tourelle de la caméra. Ce dispositif permet de maintenir constante la distance de la trompe à l'objectif, et d'éliminer les mouvements dus à la respiration. La mise au point est réglée de manière à ce que l'image soit environ le I/3 de l'objet. Nous avons utilisé un téléobjectif de $75 \mathrm{~mm}$ Angénieux monté sur une caméra Webo M. L'éclairage est fourni par une lampe basse tension à faisceau parallèle avec filtre anticalorique.

A la fin des observations, la place occupée par les œufs à l'intérieur de chacue trompe est soigneusement notée, les organes replacés in silu et l'ouverture médio-ventrale recousue. On abat les animaux de I heure à I $7 \mathrm{~h}$. 30 après la fin des observations.

\section{Isthme.}

Chez des brebis ayant ovulé depuis 2 jours, 2 jours $1 / 2$ et 3 jours, on introduit dans les trompes utérines, en passant par le pavillon, une particule de matière plastique noire que l'on pousse jusqu'à l'endroit désiré. Après suturation et réveil, l'animal est abattu dans les 45 minutes à 3 heures qui suivent la fin de l'opération.

\section{B) Études in vitro.}

L'isthme, un $\mathrm{cm}$ d'ampoule et un $\mathrm{cm}$ de corne sont isolés après abattage. On introduit dans la partie ampoulaire supérieure une petite particule de matière plastique noire de la taille d'un 
œuf, et on place aussitôt l'isthme dans une cuve à faces parallèles contenant du Locke oxygéné. Nous utilisons généralement la trompe située du côté où s'est produite l'ovulation.

L'extrémité ampoulaire de l'isthme est reliée au bras d'un levier très léger qui inscrit les contractions sur un cylindre enregistreur, tandis que l'autre extrémité sectionnée de l'organe est lestée par une bille de verre mobile au fond de la cuve.

Sur l'isthme, on trace à l'encre de Chine des traits de repère distants de $0,5 \mathrm{~cm}$.

La température de la cuve est maintenue à $37^{\circ} \mathrm{C}$ par deux lampes photo-flood disposées à une distance convenable avec interposition d'une vitre anticalorique comme le montre la figure $\mathrm{I}$. Un ventilateur placé latéralement permet un brassage convenable de l'air autour de la cuve pour éviter des échauffements localisés.

Les mouvements de l'isthme et de la particule colorée sont filmés par la caméra située à une distance telle que le rapport image /objet soit égal à 3. La caméra est placée sur un pied télesco-

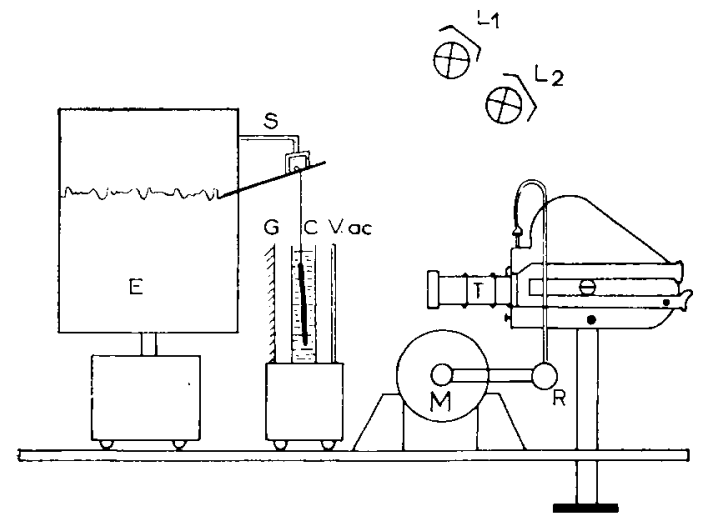

FIG. I - Schéma du dispositif d'enregistrement in vitro de la descente d'une paritcule de nalière plastique noive dans l'islhme:

V. ac : vitre anticalorique. $-\mathrm{T}:$ téléobjectif. $-\mathrm{M}$ : moteur. $-\mathrm{R}$ : came. $-\mathrm{G}:$ miroir. $-\mathrm{E}$ : cylindre enregistreur. $-\mathrm{S}$ : levier. $-\mathrm{I}_{1} \mathrm{I}_{2}$ : lampes photo-flood.

pique de manière à pouvoir suivre, parallèlement à l'axe de l'isthme la descente de la particule. Les mouvements de l'isthme ne sont pas analysables à l'œeil nu, leur rythme étant trop lent, nous avons essayé de l'accélérer en ralentissant la vitesse des prises de vues à I 50 images/minute, par l'intermédaire d'un moteur avec came agissant sur le déclencheur de la caméra. D'autre part l'analyse détaillée des films et des mouvements dont ils font état a pu être faite grâce à un projecteur dont le déroulement est actionné à la main, ce qui permet de faire varier les vitesses de projection et de suivre image par image le phénomène étudié.

La transparence de l'isthme a été améliorée par un miroir placé derrière la cuve à faces parallèles. L'isthme n'a pas été filmé constamment mais seulement pendant les déplacements de la particule, tandis que le début et la fin du fonctionnement de la caméra ont été signalés sur le tracé correspondant du cylindre enregistreur.

Dans une autre série d'expériences, la particule a été remplacée par une suspension de carbone dans du Locke remplissant la totalité de l'isthme alors ligaturé à ses deux extrémités; les traits de repère sur l'organe sont remplacés par une échelle graduée en $1 / 2 \mathrm{~cm}$, placée derrière et visible sur le film.

\section{RÉSULTATS}

\section{A. Ampoule}

\section{I. Étude globale de descente partielle.}

Comme nous l'avons exposé précédemment, toutes les études faites sur l'ampoule ont dû être poursuivies chez l'animal vivant. En effet, des expériences préliminaires poursuivies sur des ampoules excitées maintenues en survie physiologique nous ont 
révélé que, dans ces conditions, aucun mouvement ne peut être décelé sur l'ampoule. Ces résultats sont opposés à ce qu'on obtient sur l'isthme qui resteactif en culture in vitro.

a) Dans une première série d'expériences, nous avons placé une particule de matière plastique dans l'une des ampoules d'une brebis anesthésiée, et abattu l'animal 27 à 42 heures plus tard. Le tableau I montre que la particule a progressé seulement dans 2 cas sur 6 ; dans tous les autres cas, elle est demeurée à sa position initiale.

TABLEAU I

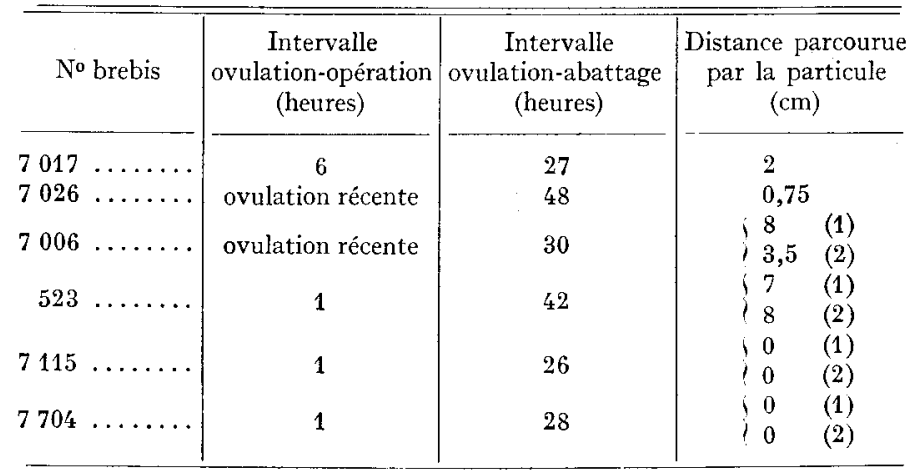

Descente d'une particule de matière plastique noire à l'intérieur de l'ampoule in vivo.

(1) Corne droite.

(2) Corne gauche.

b) En répétant cette même expérience avec une macrospore de Sélaginelle, nous n'avons observé que 2 fois des descentes importantes (tableau 2), mais dans presque tous les cas la spore de Sélaginelle a parcouru un chemin non négligeable.

TABIFAU 2

\begin{tabular}{|c|c|c|c|}
\hline No brebis & $\begin{array}{c}\text { Intervalle } \\
\text { ovulation-opération } \\
\text { (heures) }\end{array}$ & $\begin{array}{c}\text { Intervalle } \\
\text { ovulation-abattage } \\
\text { (heures) }\end{array}$ & $\begin{array}{c}\text { Distance parcourue } \\
\text { par la macrospore } \\
(\mathrm{cm})\end{array}$ \\
\hline $976 \ldots$ & 1 & 28 & 0,75 \\
\hline $1307 \ldots$ & $\begin{array}{l}\text { ovulation très } \\
\text { récente }\end{array}$ & 27 & $\begin{cases}8,5 & (2) \\
1 & (1)\end{cases}$ \\
\hline $7096 \ldots$ & 1 & 32 & $\begin{cases}1 & (1) \\
6 & (2)\end{cases}$ \\
\hline $2375 \ldots$ & $\begin{array}{l}\text { ovulation très } \\
\text { récente }\end{array}$ & 33 & $\begin{cases}2,5 & (1) \\
0 & (2)\end{cases}$ \\
\hline $7039 \ldots$ & 2 & 30 & $\begin{cases}4 & (1) \\
3 & (2)\end{cases}$ \\
\hline
\end{tabular}

Descente d'une macrospore de Sélaginelle à l'intérieur de l'ampoule " in vivo".

(1) corne gauche.

(2) corne droite.

c) Il semble dans ces conditions que l'aspect extérieur du corps puisse jouer un rôle dans sa vitesse de progression. Comme on sait qu'au moment de l'ovulation les 
œufs sont émis entourés des cellules de la corona, nous avons placé, dans les trompes d'une brebis, des paquets d'ovocytes vierges de lapine, entourés des cellules de corona radiata, liées entre elles (tableau 3 ). On assiste, même avec des temps relativement courts, à une progression importante des aufs. Certes, la préhension et le maintien transitoire de la trompe hors de l'organisme provoquent souvent un odème qui empêche le déplacement des cufs étrangers de lapine ou seulement les ralentit du côté opéré par rapport au côté témoin replacé in situ. Cependant, dans ces conditions, on constate une bien meilleure progression des œufs de lapine entourés de leurs cellules de corona que des macrospores de Sélaginelle ou des particules de matière plastique.

TABLEAU 3

\begin{tabular}{|c|c|c|c|c|}
\hline \multirow{2}{*}{ No brebis } & \multirow{2}{*}{$\begin{array}{c}\text { Intervalle } \\
\text { ovulation-opération } \\
\text { (heures) }\end{array}$} & \multirow{2}{*}{$\begin{array}{c}\text { Intervalle fin } \\
\text { d'opération-abattage } \\
\text { (heures) }\end{array}$} & \multicolumn{2}{|c|}{$\begin{array}{l}\text { Distance parcourue par les oufs étrangers } \\
\text { (cm) }\end{array}$} \\
\hline & & & Trompe observée & Trompe témoin \\
\hline $8604 \ldots \ldots \ldots \ldots$ & avant & $31 / 2$ & 0,5 & 9 \\
\hline $532 \ldots \ldots \ldots \ldots$ & avant & 1 & cdème & 5,5 \\
\hline $8610 \ldots \ldots \ldots \ldots$ & avant & 1 & B P & 1 \\
\hline $8580 \ldots \ldots \ldots \ldots$ & $1 / 2$ & 6 & 1,5 cedème & 9 \\
\hline $8703 \ldots \ldots \ldots \ldots$ & 1 & 17 & 6 & 13 \\
\hline $600 \ldots \ldots \ldots \ldots$ & 1 & 4 & $\mathrm{~B} P$ & 5 \\
\hline $7845 \ldots \ldots \ldots \ldots$ & $21 / 2$ & $31 / 2$ & 4 & $\mathrm{EC}$ \\
\hline $8614 \ldots \ldots \ldots \ldots$ & $21 / 2$ & $43 / 4$ & 0 cedème & 7 \\
\hline $7235 \ldots \ldots \ldots \ldots$ & 3 & 3 & 8 & 一 \\
\hline $624 \ldots \ldots \ldots \ldots$ & $51 / 2$ & $171 / 2$ & 6 & 5 \\
\hline $7134 \ldots \ldots \ldots \ldots$ & 6 & 16 & B M & 3 \\
\hline $697 \ldots \ldots \ldots \ldots$ & 6 & $161 / 2$ & 10 & 10 \\
\hline $690 \ldots \ldots \ldots \ldots$ & 16 & 6 & 6 & 3 \\
\hline
\end{tabular}

Descente d'un " plug " d'œufs de lapine à l'intérieur de l'ampoule in vivo.

\section{Etude de la traversée de l'ampoule.}

\section{a) Rôle des mouvements ciliaires}

Si la descente de l'œuf était assurée par des mouvements ciliaires ou par des courants liquides créés par ces mouvements, des particules de matière plastique ou des œufs de Sélaginelle devraient descendre plus facilement que des œufs de lapine entourés des cellules de la corona radiata. Quand nous avons remplacé dans les trompes de brebis, les ovocytes de lapine précédemment utilisés par une suspension du bleu de Prusse (BP), ou d'encre de Chine (E C), nous n'avons assisté à aucune progression de ces colorants. Ces résultats négatifs infirmeraient l'existence d'un courant liquide orienté.

\section{b) Rôle des contractions}

Pendant ces expériences, nous avons pu observer de petites contractions des ampoules suivies de la progression des oufs.

Nous avons donc entrepris une nouvelle série d'expériences pour essayer d'ana- 
lyser la motilité de l'ampoule en filmant les déplacements des oufs étrangers, et ceci en changeant le moins possible la position des trompes. Comme les contractions se produisent à un rythme assez lent, nous avons filmé les parties supérieure, moyenne et inférieure de l'ampoule à différentes cadences de prises de vue. Les seules observations possibles ont été obtenues avec des films pris à la vitesse de 150 images/minute. Ceux-ci nous révèlent les faits suivants :

- Il existe des contractions dirigées de la partie ovarienne vers l'isthme.

- Ce péristaltisme est de faible amplitude.

- Il est localisé à certaines zones de l'ampoule qui ne paraissent pas associés à la présence des œufs.

- Ce n'est généralement pas la même contraction qui parcourt toute l'ampoule de haut en bas.

Cette activité ne paraît dépendre ni du lieu del'ampoule considérée, ni du moment de 1'ovulation, les mouvements de 1'ampoule restant les mêmes avant et après l'ovulation, comme le montre le tableau 4 .

TABIEAU 4

\begin{tabular}{|c|c|c|c|}
\hline No brebis & $\begin{array}{c}\text { Intervalle } \\
\text { ovulation-opération } \\
\text { (heures) }\end{array}$ & $\begin{array}{c}\text { Place des ovocytes } \\
\text { dans l'amponle }\end{array}$ & $\begin{array}{l}\text { Activité de la } \\
\text { trompe }\end{array}$ \\
\hline $\begin{array}{l}7252 \ldots \ldots \ldots \\
9061 \ldots \ldots \ldots\end{array}$ & $\begin{array}{c}\text { ovulation récente } \\
5\end{array}$ & $\begin{array}{l}\text { 1/2 supérieure } \\
\text { milicu }\end{array}$ & $\begin{array}{l}\text { activité minime } \\
\text { contractions fortes } \\
\text { en certains points }\end{array}$ \\
\hline $9158 \ldots \ldots \ldots$ & 5 & 1/2 supérieure & fortes contractions \\
\hline $9401 \ldots \ldots \ldots$ & $51 / 2$ & $1 / 2$ supérieure & fortes contractions \\
\hline $9437 \ldots \ldots \ldots$ & 8 & 1/2 inférieure & activité moyenne \\
\hline $659 \ldots \ldots \ldots$ & $151 / 2$ & milicu & activité moyenne \\
\hline $7811 \ldots \ldots \ldots$ & 16 & 1/2 inférieure & activité moyenne \\
\hline 7950 . & 16 & 1/2 supérieure & activité moyenne \\
\hline $9199 \ldots$ & 16 & 1/2 supéricure & activité moyenne \\
\hline $341,1 \ldots \ldots \ldots$ & 16 & 1/2 inférieure & fortes contractions \\
\hline
\end{tabular}

Activité de l'ampoule "in vivo ", contenant un "plug " d'ocufs de lapine.

\section{En résumé.}

La progression des oufs, et des cellules de la corona radiata qui les entourent, serait due à un péristaltisme de l'ampoule, pratiquement invisible à l'œil nu, plutôt qu'aux mouvements ciliaires.

\section{B. Isthme}

\section{I. - Étude in vivo.}

Des expériences analogues à celles entreprises sur l'ampoule, où l'on introduit une particule de matière plastique noire que l'on pousse de proche en proche jusqu'au lieu d’observation désiré, nous ont donné des résultats diamétralement opposés aux précédents.

Le tableau 5 nous montre que c'est seulement à partir de 3 jours après l'ovulation, qu'une particule placée dans l'isthme a des chances de le franchir rapidement et 
régulièrement. Contrairement à ce qui se passe dans l'ampoule, la sphère de matière plastique peut donc être entraînée comme un œuf.

TABIEAU 5

\begin{tabular}{|c|c|c|c|c|c|}
\hline \multirow{3}{*}{$\begin{array}{l}\text { Temps après } \\
\text { l'ovulation }\end{array}$} & \multirow{3}{*}{$\begin{array}{c}\text { T'emps } \\
\text { d'abattage }\end{array}$} & \multicolumn{4}{|c|}{ Nombre de particules arrivées dans l'utérus on en bas de l'isthme } \\
\hline & & \multicolumn{2}{|c|}{$\begin{array}{l}\text { placées daus l'isthme } \\
\text { à } 2 \mathrm{~cm} \text { dı sommet }\end{array}$} & \multicolumn{2}{|c|}{$\begin{array}{c}\text { placces en bas de } \\
\text { l'amp̣oule }\end{array}$} \\
\hline & & nombre & $\%$ & nombre & $\%$ \\
\hline 2 jours & $\begin{array}{l}1 \mathrm{~h} 30 \\
3 \mathrm{~h}\end{array}$ & $3 \operatorname{sur} 12$ & 25 & 2 sur 5 & 40 \\
\hline 2 jours $1 / 2 \ldots$ & $45 \mathrm{mn}$ & $2 \operatorname{sur} 10$ & 20 & $2 \operatorname{sur} 10$ & 20 \\
\hline 3 jours ...... & 15 à $30 \mathrm{mn}$ & 8 sur $1 t_{1}$ & 57 & & \\
\hline
\end{tabular}

\section{II. - Étude in vitro.}

Compte tenu des résultats précédentş, ces études ont été uniquement faites en employant une particule de matière plastique noire qui était placée initialement en haut de l'isthme mais pouvait être poussée en cours d'expérience vers le milieu ou vers la partie inférieure de cet organe, à l'aide d'une petite baguette.

Nous avons étudié les mouvements musculaires de l'isthme sur des brebis prises à des stades différents après l'ovulation (de quelques heures jusqu'à 4 jours) ; sur des brebis en anœestrus ; sur des brebis castrées et soumises à un traitement hormonal.

\section{a) Isthme orienté}

Dans la cuve à survie, l'organe a été placé l'extrémité ampoulaire en haut et l'extrémité utérine en bas.

I $^{\mathrm{O}}$ Une à cinq heures après l'ovulation (3 brebis). - La particule ne progresse pas et n'effectue que de petits mouvements alternatifs de bas en haut, sur place, en se maintenant au même niveau. L'activité la plus nette de l'isthme consiste en des élongations n'intéressant que des courts segments.

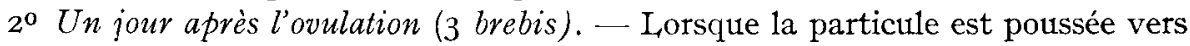
le milieu de l'isthme les contractions antipéristaltiques la ramènent rapidement en haut. La particule ne progresse guère plus que précédemment. Dans un cas cependant, poussée vers le milieu de l'isthme elle descend de $3 \mathrm{~cm}$ et poussée plus bas, franchit seule la jonction isthme-corne utérine. On décèle sur l'isthme des ondes d'antipéristaltisme (graphique I).

$3^{\circ}$ Deux jours après l'ovulation (2 brebis). - Les déplacements de la particule se limitent toujours à des mouvements de va-et-vient.

Un antipéristaltisme de la partie supérieure de l'isthme s'oppose toujours au passage de la particule. Seules des progressions de 2 à $3 \mathrm{~cm}$ ont été observées en poussant la particule vers le milieu. 


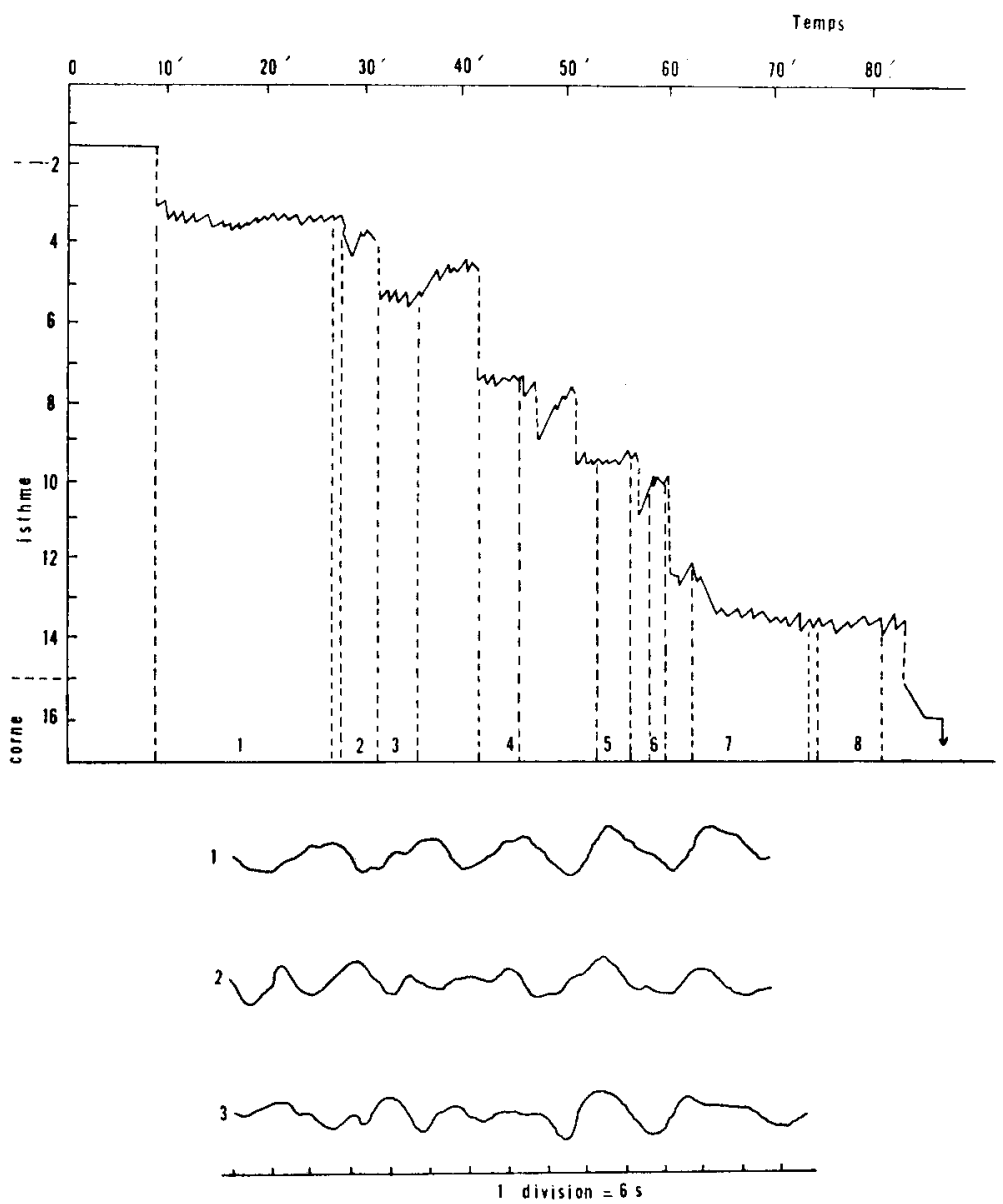

Graphique I. - En dehors des rappels à l'abscisse délimitant, sur le graphique de la progression de la particule, les séquences de prises de vues, les lignes pointillées verticales représentent les distances effectuées par la sphère de matière plastique lorsqu'elle est poussée par l'opérateur.

$4^{\circ}$ Deux jours et demi après l'ovulation (6 brebis). - Après des mouvements de va-et-vient plus ou moins prolongés au sommet de l'isthme, la particule le traverse en des temps variant de 6 à $45 \mathrm{mn}$, puis sort par l'extrémité ouverte de la corne utérine.

L'activité de l'isthme est à ce stade nettement plus importante, des ondes péristaltiques parcourent toute sa longueur et, dirigées de l'ampoule vers l'utérus, sont à l'origine de la propulsion de la particule, qui peut être très rapide.

I es contractions antipéristaltiques sont beaucoup moins énergiques (graphique 2.)

$5^{\circ}$ Trois jours après l'ovulation ( $\mathrm{I}_{5}$ brebis). - L a particule, après des va-etvient plus ou moins nombreux, franchit l'isthme en 15 à $35 \mathrm{mn}$. Les graphiques des trajets effectués sont assez semblables à ceux relevés à 2 jours et demi.

L'activité de l'isthme est alors importante, on trouve à peu près tous les différents types de mouvements enregistrés jusqu'ici : torsion, constrictions, élongations, péristaltisme et antipéristaltisme, qui peuvent du reste, alternativement, intéresser le même segment (graphiques 3 et 4 ). 
$6^{\circ}$ Trois jours et demi et quatre jours après l'ovulation. — Chez 4 brebis abattues 3 jours et demi après 1'ovulation et 3 autres, 4 jours après l'ovulation nous n'avons obtenu que dans 2 cas une descente appréciable. Dans tous les autres cas, la particule n'effectue que de petits déplacements. L'activité de l'isthme ne semble pas être comparable à celle enregistrée à 2 jours et demi et 3 jours, elle consiste surtout en constrictions et en mouvements de torsion de la trompe.

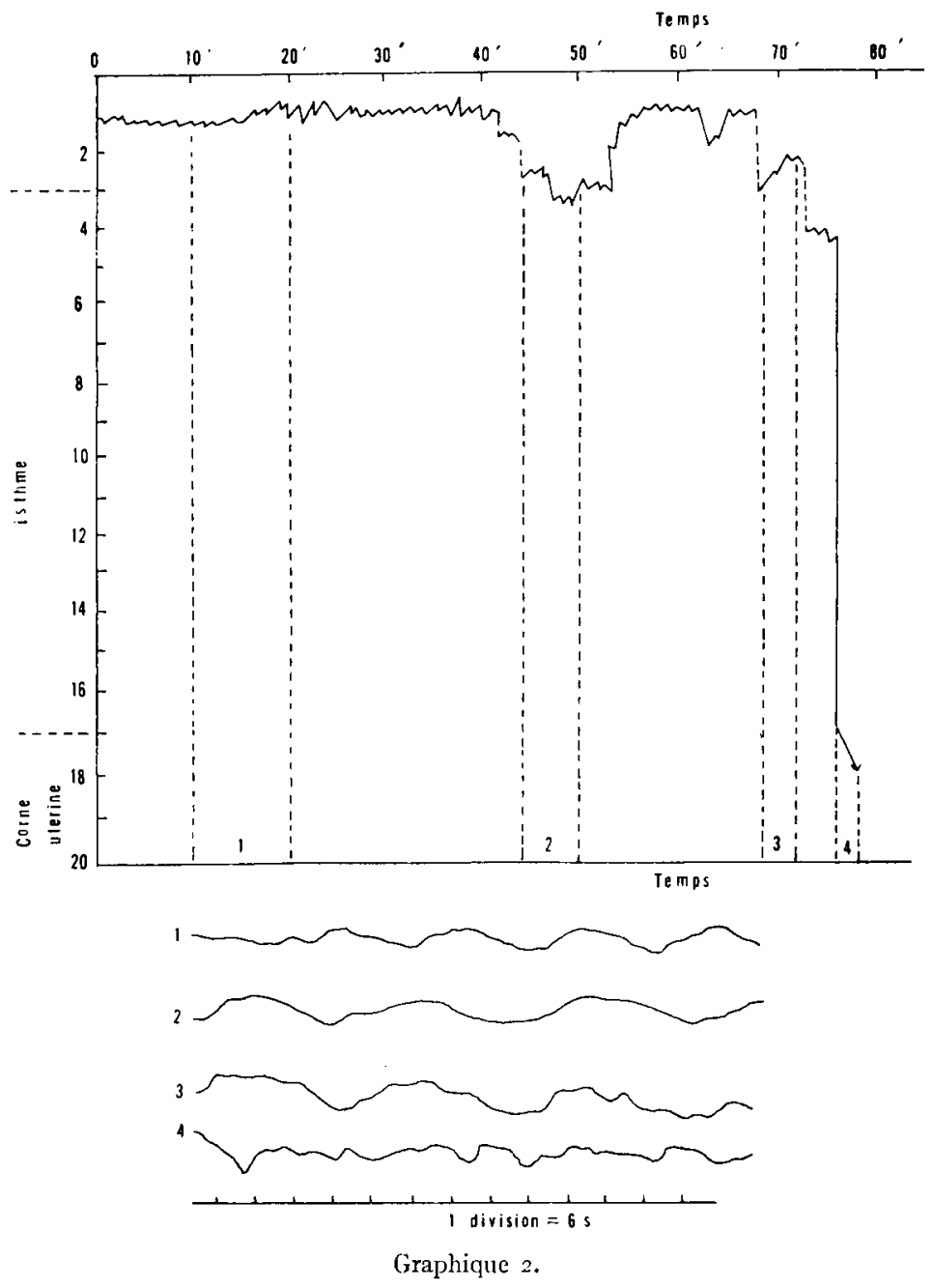

$7^{\circ}$ Brebis en ancestrus. - De la série des résultats précédents semble se dégager le fait que les mouvements de 1'isthme, qui permettent à une particule de le traverser, n'ont lieu qu'en présence d'un corps jaune âgé de 2 jours et demi à 3 jours ; en dehors de ce délai, 1 'isthme a sans doute une activité, mais une activité non orientée ou mal orientée pour assurer le déplacement des œufs vers l'utérus. Pour vérifier cette constatation, nous avons fait une expérience sur 6 brebis en anoestrus réel c'est-à-dire ne présentant pas de corps jaune, même ancien. 

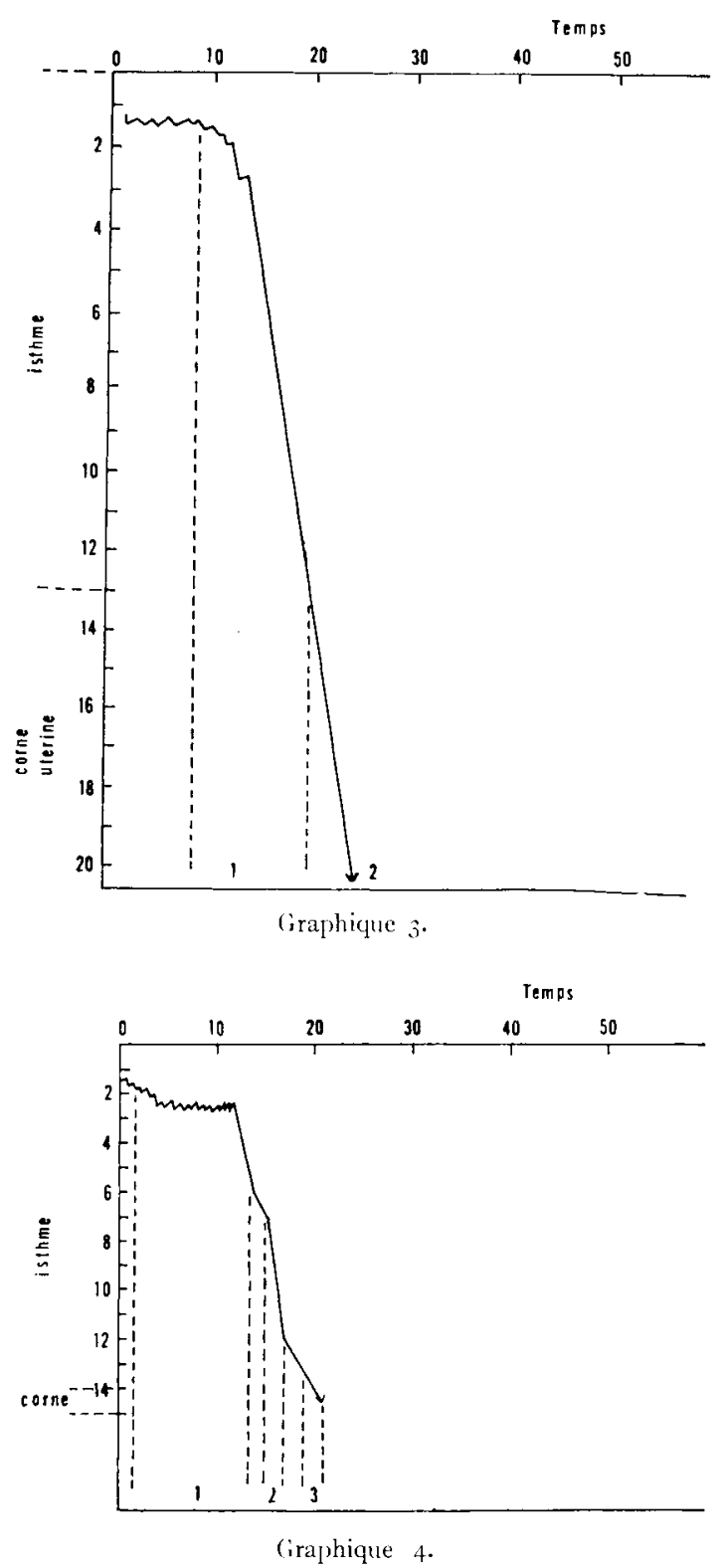

On constate que la particule se déplace très peu, elle est refoulée au sommet de l'isthme par une zone d'antipéristaltisme. Si on la pousse pour lui faire franchir cet obstacle, elle reste sur place ou à tendance à remonter ; dans deux cas set1lement nous l'avons vue effectuer une descente libre de 2 à $3 \mathrm{~cm}$ à la partie inférieure de l'isthme et sortir par la corne. L'activité de l'isthme est assez réduite et semble être surtout localisée à la moitié supérieure avec antipéristaltisme dominant et torsion tandis qu'à la moitié inférieure dominent les élongations. 
$8^{\circ}$ Brebis castrées. - La castration supprime également toute activité de l'isthme aucune particule ne pouvant alors le parcourir. Un traitement combiné oestrogèneprogestérone ne le rétablit pas, il faut procéder à une injection de $0,1 \mathrm{mg}$ de benzoate d'œestradiol déclanchant l'oestrus suivie pendant deux jours de $5 \mathrm{mg}$ de progestérone par jour pour que s'établisse une activité qui permette à une particule le franchissement.

Ceci confirme le fait maintenant classique que l'injection d'œestrogène provoque le blocage des œufs dans les trompes (Burdick, EMmerson, et WHITNEY, I940) mais non leur admission dans l'isthme.

\section{b) Isthme inversé}

Dans la cuve à survie, l'organe a été placé l'extrémité utérine en haut.

I' Deux jours après l'ovulation (4 Brebis). - La particule ne franchit pas seule le sommet de l'isthme mais peut cependant atteindre la corne utérine si on la pousse suffisamment loin dans l'isthme. Ceci est en accord avec les résultats trouvés dans la première série d'expériences lorsque l'isthme est en position " normale ». L'activité de la trompe est alors comparable à celle enregistrée précédemment.

$2^{\circ}$ Deux jours et demi après l'ovulation (2 Brebis). - L a particule peut effectuer une longue remontée et arriver dans la corne, un antipéristaltisme situé au sommet de l'isthme fait " hésiter " la particule.

$3^{\circ}$ Trois jours après l'ovulation ( 3 Brebis). - Comme dans la première série d'expériences on assiste soit à une remontée directe de la particule soit à une remontée progressive vers la corne.

Cette correspondance des trajets suivis par la particule dans les 2 séries d'expériences démontre bien que l'on ne peut faire jouer aucun rôle à la pesanteur dans le mouvement de la particule, dans notre premier groupe d'expériences et qu'il s'agit uniquement de l'influence des contractions musculaires (graphique 5).

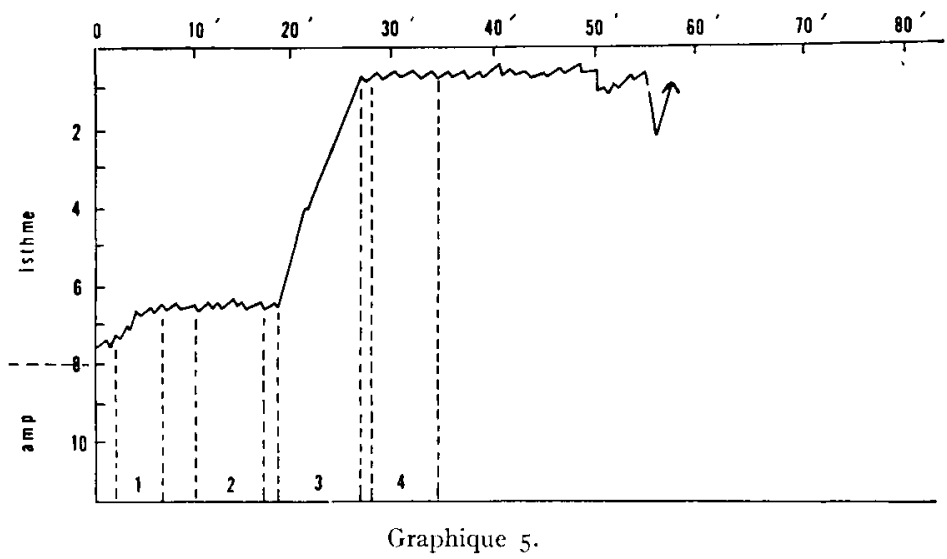

\section{II. - Nature des pulsations de l'isthme.}

Nous avons complété cette étude des mouvements musculaires de l'isthme en injectant dans la lumière une quantité juste sufflsante de carbone en stuspension dans le Locke pour mettre en évidence la lumière, sans toutefois distendre l'isthme. 
L'enregistrement cinématographique a été effectué de plus loin de manière que l'image couvre la totalité de l'isthme.

L'étude a été faite sur des brebis deux jours après l'ovulation, l'activité de l'isthme commençant alors à être plus importante que dans les premières heures qui suivent l'ovulation.

La lumière de l'isthme n'apparaît pas comme un canal continu mais comme une série de renflements séparés par des parties à lumière réduite. Ces rétrécissements localisés sont dûs à des constrictions qui évoluent de plusieurs façons :

- la constriction disparaît en commençant par l'extrémité située près de l'ampoule, la lumière s'ouvte progressivement et se referme en partant du sommet. Elle imprime au carbone un courant dirigé vers l'utérus, qui est suivi d'une remontée, quand la constriction se referme; ce mouvement explique les " hésitations " de la particule ; lorsque la constriction s'ouvre jusqu'au ventre suivant, la particule effectue une descente limitée.

- la constriction, au lieu d'être localisée, se déplace sur tout une partie de l'isthme, constituant un mouvement péristaltique.

Dans les déplacements de la particule ou de l'œuf, ce sont les mouvements peristaltiques qui sont les plus efficaces. Nous avons vu que l'existence d'ondes antipéristaltiques est très accusée dans les deux jours qui suivent 1'ovulation, comme en rend compte l'enregistrement $n^{0}$ I où la répétition de périodes semblables est la manifestation de ces ondes. Deux jours et demi après l'ovulation les ondes péristaltiques favorisant le passage de l'œuf sont également visibles sur l'enregistrement $n^{\circ} 2$.

\section{CONCLUSIONS}

Il semble que l'on puisse conclure de ces recherches que les différents types de contractions musculaires dont l'ampoule et l'isthme sont le siège permettent d'expliquer aisément les différentes étapes du cheminement de l'œuf dans l'oviducte.

Les contractions péristaltiques de l'ampoule, de faible amplitude, ne sont pas capables de mouvoir des particules de plastique de même taille que les œufs mais seulement les ovocytes eux-mêmes entourés de leurs cellules coronaires. Certes on sait que chez les Ruminants l'ovocyte perd très vite son enveloppe cellulaire mais aussitôt après l'ovulation pendant le début de la progression il en est encore au moins partiellement entouré.

Les mouvements ciliaires n'expliquent pas la progression des ovocytes dans l'ampoule après l'ovulation puisque nous n'avons jamais observé de déplacement dans l'ampoule in vitro alors que les cils continuent à battre. Ils peuvent toutefois agir sur des corpuscules de très faible diamètre puisque des auteurs ont signalé que des particules placées dans l'abdomen étaient retrouvées dans le tractus génital.

L'existence de courants liquides a également été invoquée, il semble bien que de tels courants ne puissent être en cause puisque 1'absorption des sécrétions tubaires a lieu par les franges du pavillon et non par élimination vers l'utérus ; la ligature de la partie terminale de la trompe conduisant à un gonflement considérable de celle-ci par suite d'un arrêt de la résorption liquide.

Dans l'isthme, nous avons montré l'existence de contractions musculaires antipéristaltiques puissantes pendant les 60 heures qui suivent l'ovulation. Ces contrac- 
tions maintiennent les œufs à la limite de l'ampoule et de l'isthme pendant environ 48 heures en particulier pour l'isthme, la concordance absolue des faits enregistrés in vitro avec ceux observés in vivo, nous permet de penser que les mécanismes musculaires décrits sont bien ceux qui sont en cause in vivo. Puis ces contractions s'affaiblissent et les mouvements péristaltiques peuvent alors aisément assurer le transit de l'œuf vers l'utérus.

Reçu en juin 1960.

\section{SUMMARY}

THE MOVEMENTS OF THE FALLOPIAN TUBES AND THE DESCENT OF EGGS IN THE EWE

The motility of the Fallopian tube and its role in the passage of the developing egg was studied by means of slow motion films ( 2 to 3 frames/second).

The study carried out both in vitro and in vivo for the isthmus and only in vivo for the ampulla since most of the muscular movements disappear in the isolated ampulla.

By placing either stained oocytes or black plastic particles of the same size or even carbon in suspension in Locke's solution, in the portion of the tube to be studied, it was possible to show the existence of slow peristaltic contractions in the ampulla which ensure the advance, to the isthmus, of the freshly released oocyte still entirely or partly surrounded by its corona cells. During the 48-6o hours which follow ovulation, strong antiperistaltic movements occur in the isthmus which maintain the egg at the junction of the isthmus with the ampulla ; it is the peristaltic movements affecting either the whole or only portions of the isthmus which eventually carry the egg into the uterine horn.

The different kinds of contractions were also initiated experimentally by altering the cestrogenprogesterone equilibrium of the previously castrated ewe.

\section{RÉFÉRENCES BIBLIOGRAPHIQUES}

Bernstein F. Ferester M., I940. Estrogenic effects upon tubal contractility and the vaginal secretion in the menopause. Endocrinol., 26, 247-252.

BuRdiK H. O., EMERSON B. B., WHITNEY R., I940. Effects of testosterone propionate on pregnancy and on passage of ova through the oviduct of mice. Endocrinol., 26, 1080-1086.

Davies A. M., I948. Fallopian tubal motility in relation to the menstrual cycle. Amer. J. Obst. Gynecol., $\mathbf{5 6}, 655-66_{3}$.

DE LA I'Uente F., I95I. La motilité de la trompe de Fallope "in vitro ». Rev. Franc. Gynecol. Obst., 46, 256-64.

Dubreuil G., r946. Les trompes de Fallope chez la Femme. Vigot trère ed. Paris.

Geistes M., Salmon U. T., Mintz M., 1938. The effect of estrogenic hormone upon the contractility of the fallopian tubes. Amer. J. Obst. Gynecol., 36, 67-77.

Ortavan1 R., Thibault C., Wintenberger S., i949. Contribution à l'étude de la superovulation expérimentale chez la brebis. Ann. Endocrin., 10, I70-1 73 .

SECKINGER D. L., 1923. Spontaneous contractions of the Fallopian tubes of domestic pig with reference to the oestrus cycle. J. Hoplanis. Hosp. Bull., 34, 236.

Schling E., I958. Untersuchungen über den Eitransport bei Wiederkaüern. Verh. dtsch. Zool. Ges., Frankfurt 283-89.

WESTMAN A., I926. A contribution of the question of the transit of the ovum from ovary to uterus in rabbits. Acta Obst. Gynecol. Scand., 5, (suppl.) 7-104.

WHIMPFHEIFER S., FoRESTEN M., I939. Role of the uterus in the production of manometric fluctuations during uterotubal insufflation (Rubin test). Amer. J. Obst. Gynecol., 37, 405-4I2.

Wintenberger S., i 955. Étude du mécanisme de descente de l'œuf fécondé dans l'oviducte de brebis. Ann. Endocrinol., 16, 383-385. 